\title{
PHYSIOLOGICAL MECHANISMS UNDERLYING THE ELECTRICAL ACTIVITY OF THE BRAIN *
}

\author{
BY \\ MARY A. B. BRAZIER \\ From the Department of Neurology and Psychiatry, Harvard Medical School, and the Department of Psychiatry and \\ the Electro-encephalographic Laboratory of the Massachusetts General Hospital, Boston $\uparrow$
}

(RECEIVED JANUARY 8, 1948)

Eighteen years after the first detailed work appeared on the electrical activity of the brain(Berger, 1929) we have still no adequate understanding of its mechanism. There have been many hypotheses, and to review all the supportive data for each would be too space-consuming, but since they are described in the journals a reference list may suffice. For example, there is the evidence, based on the observed behaviour of the EEG with rising body temperature and with metabolic stimulants and depressants (Hoagland, 1936), for the dependence of the electrical rhythmicity on continuous steady-state chemical events within the cortical cells. This hypothesis attempts only an explanation of the resting rhythm, and Hoagland (1944) states that the findings should not be interpreted as meaning that the only modifiers of brain wave frequencies are necessarily changes in cell respiration. There is the hypothesis of Gerard (1941) that the EEG is a rhythmic variation of a somatic potential, demonstrated as a steady DC potential difference between the pia, where the axones end, and the area near the ventricle where cells lie (in the frog's brain). Gibbs and others (1940) suggested that the electrical activity of the cortex is a manifestation of the activity of a great number of chemical oscillators having different natural periods.

The hypothesis that interplay between the deeper centres and the cortex is responsible for the alpha rhythm grows out of the work of Bishop $(1933,1936)$, of Lorente de Nó $(1934,1935)$, and of Dusser de Barenne and McCulloch (1938). It was suggested that the cortical cells are in continuous interactivity

\footnotetext{
* Communication to the First International EEG Meeting, London, July 1947.

t Aided in part by grants from the United States Navy Department, Office of Naval Research, N5 ORI-76, Projects 8 and 14.
}

with the thalamic cells; that each thalamic neurone projects to many cortical cells, and thus, by recurrent paths through their axones, interacts with great numbers of cells in the thalamus; and that the alpha rhythm may be determined by the delay in these reverberating circuits, or in other words, by the number of synapses involved. However, evidence? has been produced by Morison and Dempseya (Morison and Dempsey, 1942; Dempsey ance Morison, 1942) that the alpha rhythm of the resting cortex is not dependent on impulses travelling along the primary projection fibres from the recognizedo thalamic nuclei, and this opens the possibility for transmission by separate neuronal pathways of non-specific afferents from undifferentiated thalamic nuclei (Dempsey and Morison, 1942; McCulloch, 1947). There are data from Fortuyn and Jasper's experiments on cats which show that rhythmic potentials in the entire cortex can be co-ordinated from a very restricted thalamic area of less than $2 \mathrm{~mm}$. in diameter (Jasper and others, 1946 ; Fortuyn and Jasper, 1947). It has been suggested (Dempsey and Morison, 1942) that this rhythm of 8 to 12 per second activity may result from constant bombardment by subliminal impulses which sum periodically in the cortex rather than a reverberation in a closed circuit.

There is reason to doubt that this mechanism operates for the frequencies faster than the alpha range, since these persist after section of the thalamic radiations (Bishop, 1933 ; Chatfield and Dempsey, 1942), and therefore presumably represent intrinsic cortical activity.

Later work by Morison threw more doubt on the dependence on reverberation involving corticothalamic connexions, since an 8-12 per second 
rhythm could be recorded from the thalamus for as long as three days after bilateral decortication (Morison and Bassett, 1945 ; Morison and others, 1943).

Most of these hypotheses have assumed as a premiss that the electrical activity in the brain travels only along an axonal or dendritic pathway, but, as Adrian (1947) has emphasized, there is the possibility that neurones may influence one another not only by impulses in neuronal pathways but by electrical field effects. Such a mechanism in the development of continued rhythmic activity has been demonstrated in isolated neurones, in the spinal cord, and in the brain.

From observations on isolated nerve preparations it has been shown that the action potential of an active portion of nerve can influence a resting portion across an imposed block (Hodgkin, 1937; Lorento de Nó, 1939 ; Blair and Erlanger, 1939), and that in certain circumstances activity in one nerve fibre can affect adjacent ones (Hoagland, 1933 ; Katz and Schmitt, 1940). Bremer's (1947) work on spinal cord discharges, in which he found that synchronized beats could be maintained in two adjacent segments although all neuronal connexion between them was severed by complete transection of the cord, supports the concept of a synchronization maintained by electrical spread in the absence of fibre connexions. And in the brain, Libet and Gerard's (1938) finding that after complete transection one half of a frog's brain could influence the beat of the other half, demonstrates a spread of excitation in the absence of any fibre connexion. These same workers (Gerard, 1941; Libet and Gerard, 1938 and 1939 ; Gerard and Libet, 1940) have demonstrated that the cortical rhythms of the frog's brain can persist when all synaptic transmission has been blocked by nicotine. In this context O'Leary's (1944) reminder that we are studying potential records from active tracts embedded in electrolytically conducting material is pertinent.

To these hypotheses must be added that of Eccles (1945) and Brooks and Eccles (1947), who have suggested that the brain potentials may result from the delicate poise between excitatory and inhibitory action in the network of Golgi cells in the brain, so that any agent tending to depress cortical activity would tip the balance towards inhibition by converting some excitors into inhibitors, whereas raised excitation would have the reverse effect.

The relative significance of these many hypotheses waits only the uncovering of more facts, and it is probable that each will be found to play some part in contributing to the final explanation. It is not suggested that experiments in human physiology are likely to give the final proof for any hypothesis of a mechanism so complex, but, since any such hypothesis needs to cover the observed data of the EEG in man, perhaps human experiment may yield evidence of a supportive nature.

\section{The Present Study}

In the present paper the results of three types of experiment on the human subject will be examined with such an object in view. The experimental data consist of studies of the effect of anoxia, of hypoglycæmia, and of pentothal anæsthesia on the wave frequencies in the electro-encephalogram of the human subject.

\section{ANOXIA}

In the course of several years' work on the effect of anoxia (or, to be more exact, of hypoxia) on man we have had plentiful opportunity to confirm the original observations of Berger (1934), and of many other workers since that time (Bremer and Thomas, 1936 ; Davis and others, 1938 ; Lennox and others, 1938), that oxygen lack causes the appearance of slow waves in the EEG. Sometimes the change in the EEG trace is visible in the record on inspection by the unaided eye, but the finer developments of this change can better be studied by automatic analysis of the wave complex, since the presence of slower frequencies may sometimes be masked by more prominent waves of higher amplitude. In the present work an electronic analyser of the type designed by Walter has been used.* For a full description of this technique the original publication should be consulted (Baldock and Walter, 1946).

The type of record obtained is seen in Fig. 1. For the sake of simplicity only the EEG trace which is being automatically analysed is reproduced here, although recordings were also made from many parts of the head by a Grass inkwriting oscillograph. The tracing illustrated was recorded from two electrodes, one on the occipital region and one on the parietal region of a normal man who had in this area a strong alpha focus which blocked to a visual stimulus. Throughout this report the alpha band will be defined as frequencies of 8 to 13 cycles per second. Below the EEG is seen the heart rate recording, and below this the frequency analysis of

\footnotetext{
* This instrument consists of two banks of 24 oscillators, each designed to resonate to a specific frequency. A resonating circuit is available for each of the usual EEG frequencies, 1.5 cycles per second to 30 cycles per second, and each resonator is connected to an integrating circuit and storage condenser. Every 10 seconds a mechanical scanner discharges these storage condensers in turn through the circuit of a recording pen which gives a deflection at each through the circuit of a recording pen which gives a deflection at each discharge, the height of the deflection being a function of the activity at that frequency. By the device of having a suitably longer pen for the analyser's trace, this can be written immediately over that part of the original EEG of which it is the analysis. As one bank of storage condensers is discharging, the second is charging, so that a continuous analysis in epochs of 10 seconds each is made possible throughout the whole period of the EEG recording.
} 
the 10-second strip of EEG shown here. The height of the peaks in the analyser's trace is a function of the amount of activity at each frequency, a very small pip (approximately equal to the height of the calibration mark for the EEG on the right of the illustration) representing the baseline for each frequency when no signal is being received. The EEG shows a slowing at the level of reduced oxygen as compared with that in air, and the analysis shows a shift of the alpha activity from a predominantly 11 and 10 cycle rhythm, through a stage at 10 and 9 after 13 minutes of low oxygen, to 8 and 9 cycles at 19 minutes and finally to 8 and 7 . It will be noticed that the heart rate also has changed, having accelerated from 66 to 90 . His capillary blood sugar level at the time of the test was $120 \mathrm{mg}$. per $100 \mathrm{c.cm}$.

Such an effect as is shown in Fig. 1 is typical when breathing for a short time a gas mixture moderately low in oxygen content. In a series of some 150 experiments on subjects examined in an anoxia chamber from which the exhaled carbon dioxide was removed, and a further series of 50 in which a mask with a one-way valve was worn for the administration of low oxygen, we have found essentially this same type of response. The latter part of this series has been examined by automatic analysis and we have been interested to study the results in the light of the various hypotheses briefly outlined at the beginning of this paper.

This gradual shift of the alpha activity, with the dominant frequency of the alpha band moving step by step to the slower side, confirms our previous results found by manual analysis, and also those of Engel and others (1944). It differs in detail from the case analysed by the method of Grass and Gibbs (1938) illustrated by Gibbs and others (1940), where the dominant peak in the alpha band remained at the same frequency (although dropping in height) as long as consciousness was maintained, while the energy in the frequencies slower than alpha increased markedly.

Of course, taking isolated moments in time such as are shown in Fig. 1 does not tell us very much about the way the slow waves have developed, and it is of interest to know whether they develop as a gradual deceleration of the normal synchronized beat, such as would be expected were the rate to have a direct relationship with the metabolic activity of cortical cells, or whether they appear as discrete slow frequencies extraneous to the alpha band originating in other, perhaps sub-cortical, cell groups and rising in voltage until they mask persistent alpha.

In Fig. 2 has been plotted the amplitude of the peak (expressed as a percentage of the maximum possible deflection) at each frequency of the analyser's trace as it was found by measurement at approximately one minute intervals throughout the whole experiment in this same subject. When plotted in this way the highest peaks, that is, the frequencies at which there is most activity, are seen to be at 11,10 , and 12 cycles per second in the baseline record, with no significant activity slower than 9 per second. The breathing of 8.5 per cent. oxygen begins at the first vertical line and continues for nearly thirty minutes to the next vertical line. As this period of anoxia progresses there is a slow fall in the 12, 11, and 10 peaks, with an initial increase in 9 cycles per second which later falls as 8 cycles per second activity increases and the peaks in the slower frequency band become more prominent. The heavy dots indicate the highest peak at each minute step, that is, the dominant frequency at that time, and if we follow this through the whole period we see it decelerate gradually from 11 cycles per second through 10 and 9 to 8 , and finally to 7 and 6 cycles per second. All activity slower than 9 cycles per second disappears with the first few breaths of air.

In Fig. 3 an experiment on another normal subjecto in the anoxia chamber has been plotted in a similar manner. The highest peaks during the baselined period in air in this case are 11,12 , and 10 with lesser peaks at 9 and 8 , and minimal activity below 8 cycles per second. The breathing of 8.5 per cent. oxygen begins at the first vertical line, and in this case continues for only fifteen minutes. As this period of anoxia progresses there is a slow fall in the $12,11,10$, and 9 cycle peaks, little change at 8 cycles, and a marked rise in the frequencies slower than 8 . The dominant frequency at each minute step (again indicated by the heavy dots) falls from 12 through the intermediate frequencies to 6 and 5 cycles per second. There was no significant activity slower than 3 cycles at any time in this record. In the last minute of anoxia there is little evidence of persistent alpha, but it returns with spectacular rapidity with the first few breaths of air, and there is a simultaneous disappearance of the slow activity.

Looked at in this way the data are suggestive of a slow deceleration of the synchronized beat of the cell group under observation rather than an eruption of slow waves among persistent alpha activity, but there are some assumptions here the validity of which need to be established by experiment. One is that the change in the relative heights of the peaks represents a change in activity. This arises from our ignorance as to whether a decrease in the prominence of the alpha band in an original EEG tracing represents a decrease in electrical 


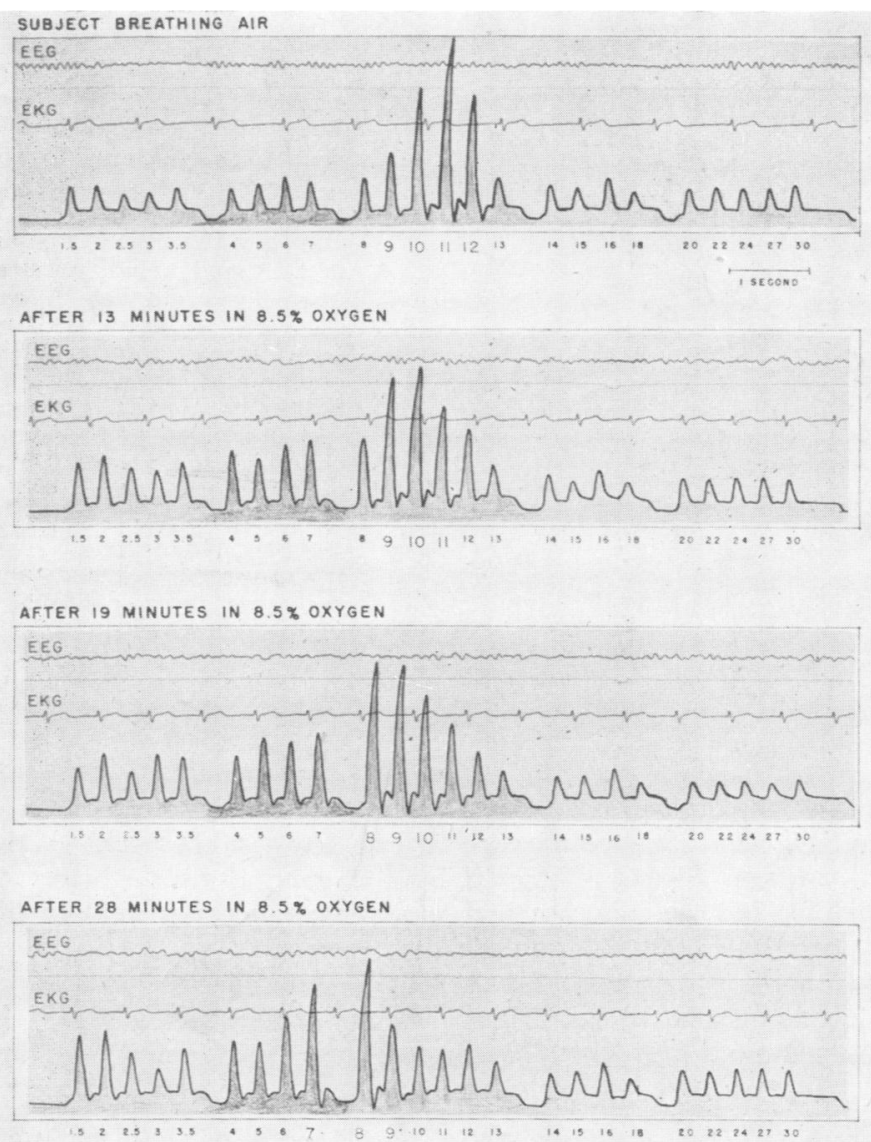

FIG. 1.-Effect of anoxia on EEG frequencies. Electroencephalograms, with simultaneous automatic analysis, recorded between two scalp electrodes, one over the parietal and the other over the occipital region of the left hemisphere of a normal man. For clarity only that EEG tracing which was being analysed is reproduced here. The second channel records the heart rate. The frequency represented by each excursion of the analyser's trace is indicated! by the number below it.

activity or whether it indicates a lesser degree of synchronization of the same amount of activity. Only experiment can decide the issue. Another question is whether the observations charted here are indices of the activity of a given synchronised cell group rather than a changing interplay of many independent sources each contributing its own frequencies to the composite picture. The view that Fig. 1 represents a deceleration of the rate of beat of a cell-group entity is supported by the fact that opening the eyes, which blocks the activity in the alpha band, produced blocking of the slower frequencies at 6,7 , and 8 cycles per second later in the experiment when the effect of anoxia was operating. This problem can be further met by making use of interchannel analysis (as described by Baldock and Walter, 1946). By this method only those potential changes which are out-of-phase in the two channels reach the analyser. By searching with electrodes on the head, a position can be found for the electrode which is common to the two channels, where the analysis shows a strong alpha rhythm, out-of-phase in the two channels and blocked by visual stimuli. Presumably such a positioning of the electrode then approximates very closely to an alpha focus. This method has been used in all the later work.

It is known that the cortex is more vulnerable to anoxia than the subcortical and phylogenetically older areas of the brain (Heymans and others, 1934 ; Sugar and Gerard, 1938) and the EEG data just discussed might well point to a slackening in metabolic rate of cortical cell respiration, but it might also indicate a decreasingly influential role of the cortex in a cortico-thalamic interplay. We have not carried any of our experiments on human subjects to an oxygen level low enough for consciousness to be lost as a result of anoxic anoxia. We have had one subject who, in a sitting position, fainted after some minutes of breathing a mixture low in oxygen although the oxygen content did not go below 10.5 per cent. and his capillary blood sugar 
level was $113 \mathrm{mg}$. per $100 \mathrm{c.cm}$. Here presumably anoxæmia produces a more sudden oxygen lack (as well as a glucose lack and accumulation of carbon dioxide) due to the insufficient blood flow, in contrast to the slowly decreasing supply of oxyhæmoglobin in the circulation in anoxic anoxia. And if loss of consciousness indicates a depression of the cortex so that thalamo-cortico-thalamic circuits are now disrupted, the EEG might be expected to record waves originating in deeper centres.

The EEG findings in this case are shown in Fig. 4. Here the more exact localization of an alpha focus was made by interchannel analysis as described above. In this experiment the recording paper was being run at half speed to save paper. Again for the sake of clarity only the original tracings of which the interchannel analysis is being made are reproduced here, although simultaneous recordings were also made from other parts of the head. As would be expected at this blood sugar level and with such a mild degree of oxygen lack, there was little change in the EEG except for some occasional 5 and 6 per second activity. At the twelfth minute there was a transient increase in the height of some of the alpha peaks and the appearance of some 4 to 7 cycle activity; at this time the subject began to show marked pallor and profuse sweating, and his pulse became weak and slow. He complained of nausea, and shortly afterwards (thirteen and a half minutes after he began to breathe the mixture low in oxygen) delta waves appeared in the record and he fainted. He recovered within one minute and the EEG promptly returned to normal.

Thus, in this record, steady activity of alpha frequency is suddenly broken by the eruption of
FIG. 2.-Progression of EEG changes in anoxia. Chart depicting the height of the peak at each frequency of the analyser's trace from 5 to $12 \mathrm{c} / \mathrm{s}$ during a period of anoxia. With the exception of four and a half minutes near the beginning of the experiment the plots have been made at approximately one-minute intervals. The period of anoxia began at the first vertical line and ended at the second. The heavy dots indicate the dominant frequencies at each given moment in time. This is the same experiment and the same electro-encephalogram as that shown in Fig. 1.

slow waves as the subject faints. This effect is seen more clearly in Fig. 5, where the height of the peaks is charted by the method used in Fig. 2. In this case the delta waves appear to replace the alpha during the short period of unconsciousness. $\stackrel{\otimes}{\varrho}$ This change in the EEG on fainting closely resembles that described by Romano and Engel (1945) in vasodepressor syncope.

\section{HYPOGLYCAMIA}

Similar methods have been applied to the study of data on subjects whose blood sugar level has been artificially lowered by insulin, since hypoglycæmia is known to affect the EEG (Berger, 1937 ; Hoagland and others, 1937 ; Lennox and others, 1938; Gibbs and others, 1940; Brazier and Finesinger, 1944; Heppenstall, 1944 ; Engel and others, 1944). From a large series of subjects so treated, Fig. 6 is representative of the effect found. In this case the electrode placements and connexions were those indicated in the illustration, with localization of an alpha focus made by interchannel analysis; the insulin dosage was 25 units.

In the case shown in Fig. 6 the occipital electrode common to the second and third channels was placed in such a position that the recorded alpha activity was out of phase between them. In this illustration the EEG is seen at three levels of blood sugar-98, 60, and $52 \mathrm{mg}$. per $100 \mathrm{c.cm}$. The shift of activity from frequencies centering round 11 cycles per second to 7 cycles is clearly seen. At the time of the second EEG sample shown here, 43 minutes after the intravenous injection of insulin, there was no sweating, no subjective sensation, and the heart rate was 88 . Sixty-three minutes after the injection the heart rat had dropped to 64 and the subject was 


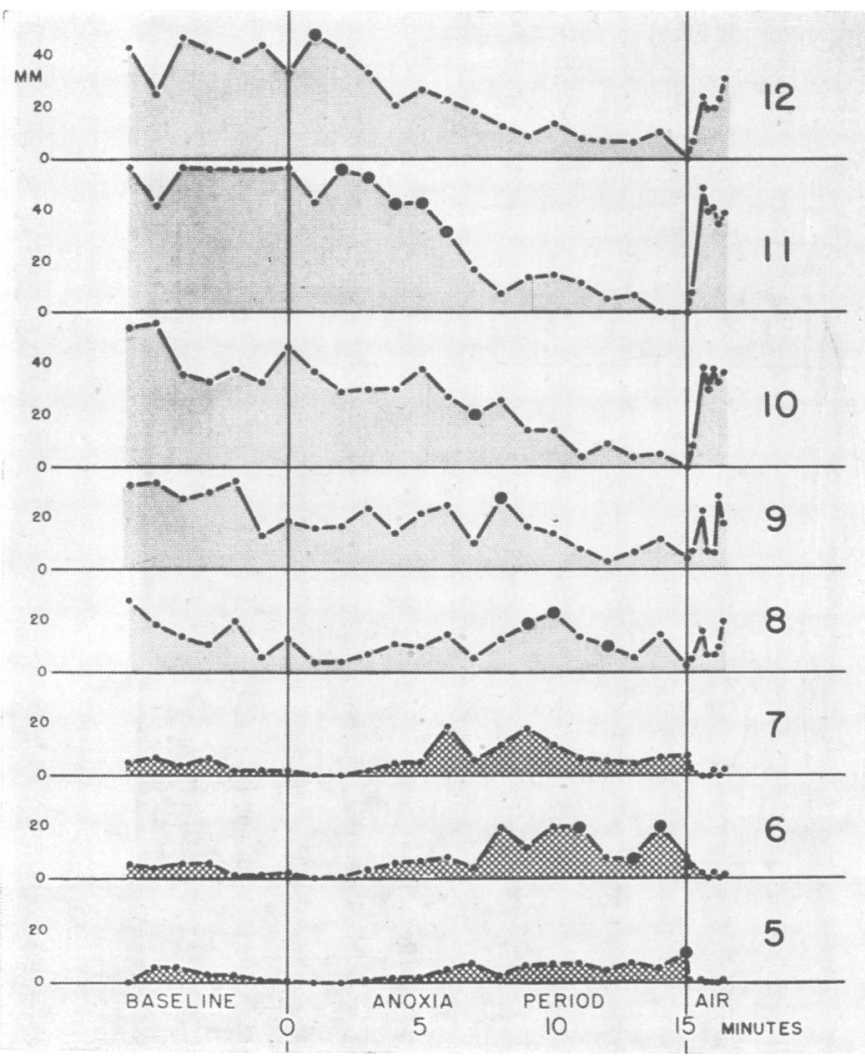

FIG. 3.-Progression of EEG changes in anoxia. Chart depicting the height from minute to minute of the peak at each frequency of the analyser's trace from 5 to $12 \mathrm{c} / \mathrm{s}$. Up to the first vertical line the subject was breathing air. In the fifteen minutes between the two vertical lines he was breathing 8.5 per cent. oxygen with 91.5 per cent. nitrogen. At the second vertical line he again breathed air. The EEG was recorded from two scalp electrodes, one over the parietal and the other over the occipital region of the left hemisphere of a normal man. There was at no time any significant activity at frequencies below $5 \mathrm{c} / \mathrm{s}$. The heavy dots indicate the dominant frequency at each given moment in time. The height of the peaks is plotted in millimetres.

sweating profusely. After this he began to recover spontaneously and the EEG also showed some improve-ment. On the intravenous injection of $50 \mathrm{~g}$. of glucose the clinical signs disappeared almost immediately but the EEG did not return so rapidly to normal.

We have found this delay in recovery of the alpha band in all our cases with mild hypoglycæmia induced by insulin : it is in sharp contrast to the behaviour of delta waves accompanying insulin coma, which disappear promptly on injection of glucose. The frequency shift in the alpha band does not bear a direct relationship to the sugar level of the circulating blood, and persists long after the autonomic disturbances have ceased. This delay in return of normal cortical activity after intravenous administration of sugar is similar to that found in dogs with insulin hypoglycæmia (Hoagland and others, 1939). It is interesting to note that the individual whose EEG is shown in Fig. 6 had fast activity in the 20 to 30 cycles per second band and that, in contrast to the alpha band, this did not slow as the blood sugar level fell.

In contrast to the effect of mild anoxia where the slowing of the EEG is accompanied by an increase in heart rate, in the insulin effect there is a slowing of the heart rate as well as of the EEG. When the data from the whole experiment are plotted by the method already used in the anoxia experiments the result, given in Fig. 7, shows the same gradual deceleration of rate. This chart also illustrates the incipient spontaneous recovery and the delayed response to glucose injection: 15 minutes after the glucose was given, there is very little activity at 11,12 , and 13 cycles per second although the blood sugar at this time was $232 \mathrm{mg}$. per $100 \mathrm{c.cm}$. Although this subject had a less regular EEG pattern than those whose records are charted in Figs. 2, 3, and 5, with more minute-to-minute variation, the general trend shows through quite clearly.

At the beginning most of the activity was at 11, 12, and 13 cycles per second. As the insulin began to take effect 9 and 10 cycles per second activity increased, until finally almost all these frequencies were replaced by 6 , 7 , and 8 cycle waves. At about seventy minutes after the injection the effect of the insulin spontaneously began to wear off, and at eighty minutes the recovery was accelerated by the intravenous injection of glucose.

This gradual shifting of the activity down the frequency range towards the slow side confirms our 


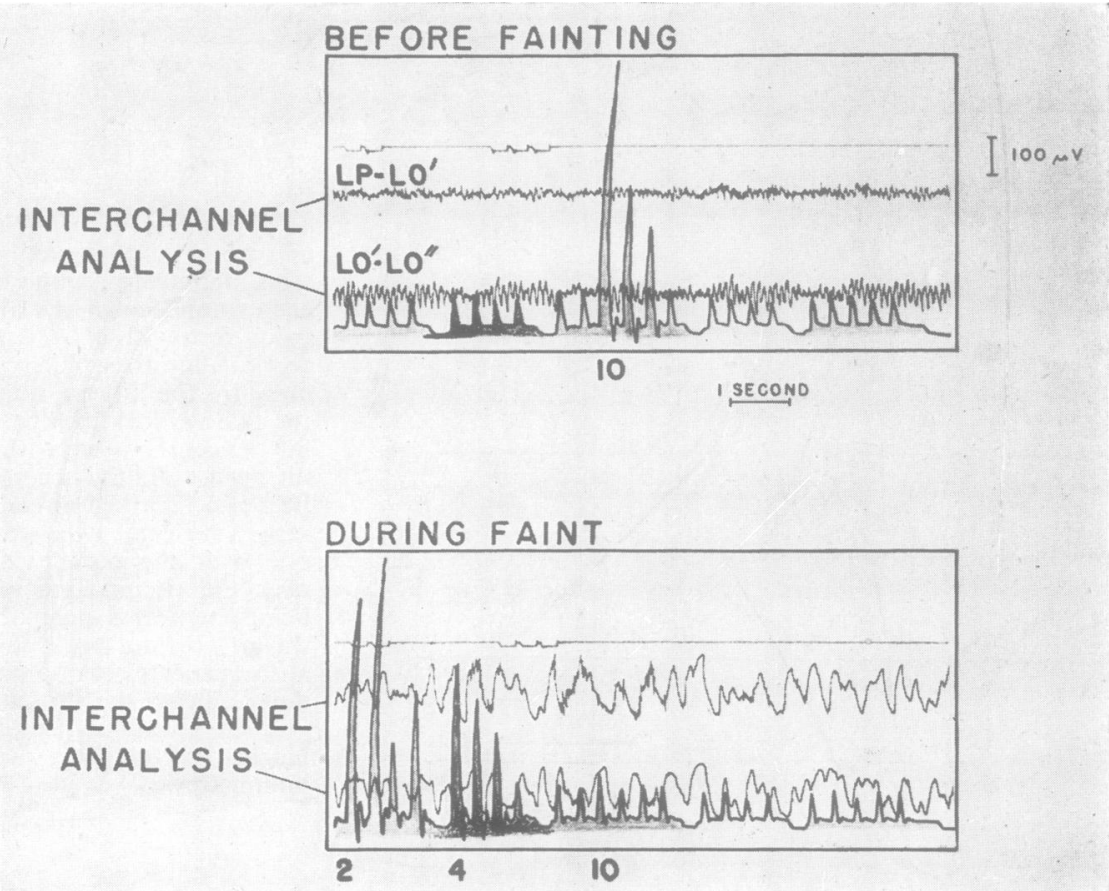

FIG. 4.-Changes in EEG during a faint. The electrode placements and connexions were those indicated in the diagram. Interchannel analysis is of the activity out-of-phase

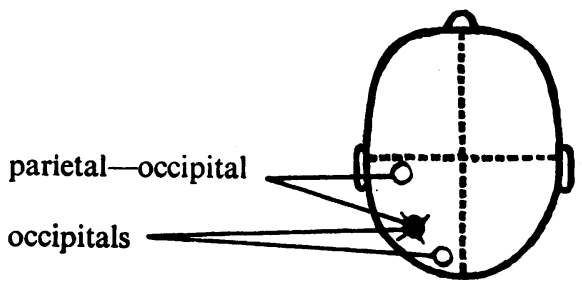
between the two channels, that is, of an alpha focus at X. For clarity only these two channels are reproduced here. Recordings were made at a paper speed of $15 \mathrm{~mm}$. per second.

The frequency of the most prominent peaks in the analyser's trace is indicated by the numbers below them. The EEG was analysed for frequencies between 2 and $30 \mathrm{c} / \mathrm{s}$.

previous findings by manual analysis (Brazier and others, 1944), and also those of Engel and others (1944), who used a different form of manual analysis. It is also in agreement with the case illustrated by Gibbs and others (1940) depicting the change found in the Grass frequency spectrum with a similarly small lowering of the blood sugar level (in their case from 105 to $74 \mathrm{mg}$. per $100 \mathrm{c.cm}$.). Their illustration shows a shift of the dominant alpha peak from 10 to 9 cycles, with a rise in the amplitude of the 8 and 7 cycle peaks. The result is, however, at variance with these authors' statement that " there is no definite shift in peaks until the sugar level falls below $29 \mathrm{mg}$. per $100 \mathrm{c.cm}$."

The mode of development of the slow activity resulting from mild anoxia and from mild hypoglycæmia is so similar that it suggests a similar underlying mechanism, one in which a cell-group entity is responsible for the effects seen. Opening the eyes has the same effect on the slow activity in hypoglycæmia as in anoxia, for the activity centering around 7 cycles per second at the low blood sugar level is blocked by opening the eyes, just as is the original alpha band before the insulin takes effect. The mode of appearance of slow waves in the record of the subject who fainted is, however, strikingly different. Here, with loss of consciousness, there is a momentary loss of all alpha frequencies and replacement by delta waves. In the case illustrated in Figs. 4 and 5 the recording was being made from an alpha focus localized by phase-reversal and interchannel analysis, and such a result suggests that the cell group responsible for the alpha activity at this focus has, on loss of 


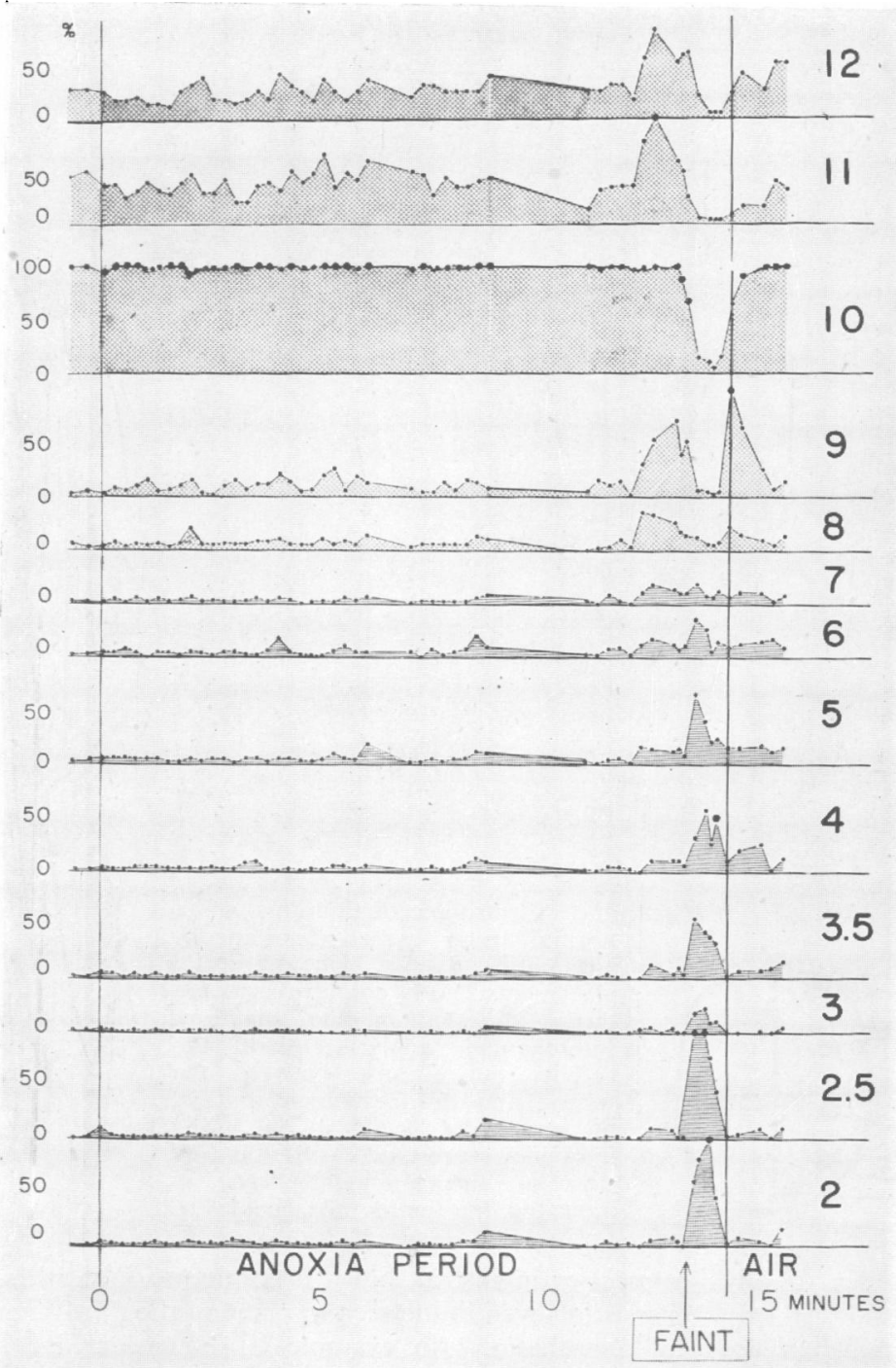

FIG. 5.-Progression of EEG changes during a faint in 10.5 per cent. oxygen. Chart showing the height of the peak at each frequency of the analyser's trace from 2 to $12 \mathrm{c} / \mathrm{s}$ in an EEG recorded during a short period of mild oxygen lack, at the end of which the subject fainted. With the exception of a two-minute period beginning at the ninth minute, during which the subject was being interrogated, the plots have been made at half-minute intervals. This is the same individual and the same electroencephalogram as that shown in Fig. 4.

consciousness, become disorganized or depressed in such a way as to release slower rhythms from deeper structures.

\section{Pentothal Anfesthesia}

We have been interested to study by similar methods of analysis the EEG changes accompanying loss of consciousness induced by anæsthetic drugs. We have in the first place used sodium pentothal, since the mode of action of barbiturates on the brain has been worked out in more chemical detail than that of many other anæsthetics.

The procedure followed has been to give the pentothal by intravenous injection of a 2 per cent. solution given at a rate calculated to induce thirdstage anæsthesia within about three minutes. In the course of an investigation on the mode of action of pentothal we have recorded electro-encephalograms in 112 experiments on 75 subjects. We have found the EEG changes to be strikingly consistent : in every case the first change is the appearance of fast activity, usually of rather high voltage, followed later by slow waves (Brazier and others, 1945). The fast activity first appears during the first stage of anæsthesia and is not seen while the subject is truly alert but only when there is some degree of clouding or euphoria. In most cases the fast waves increase in amount as the first stage of anæsthesia progresses. 


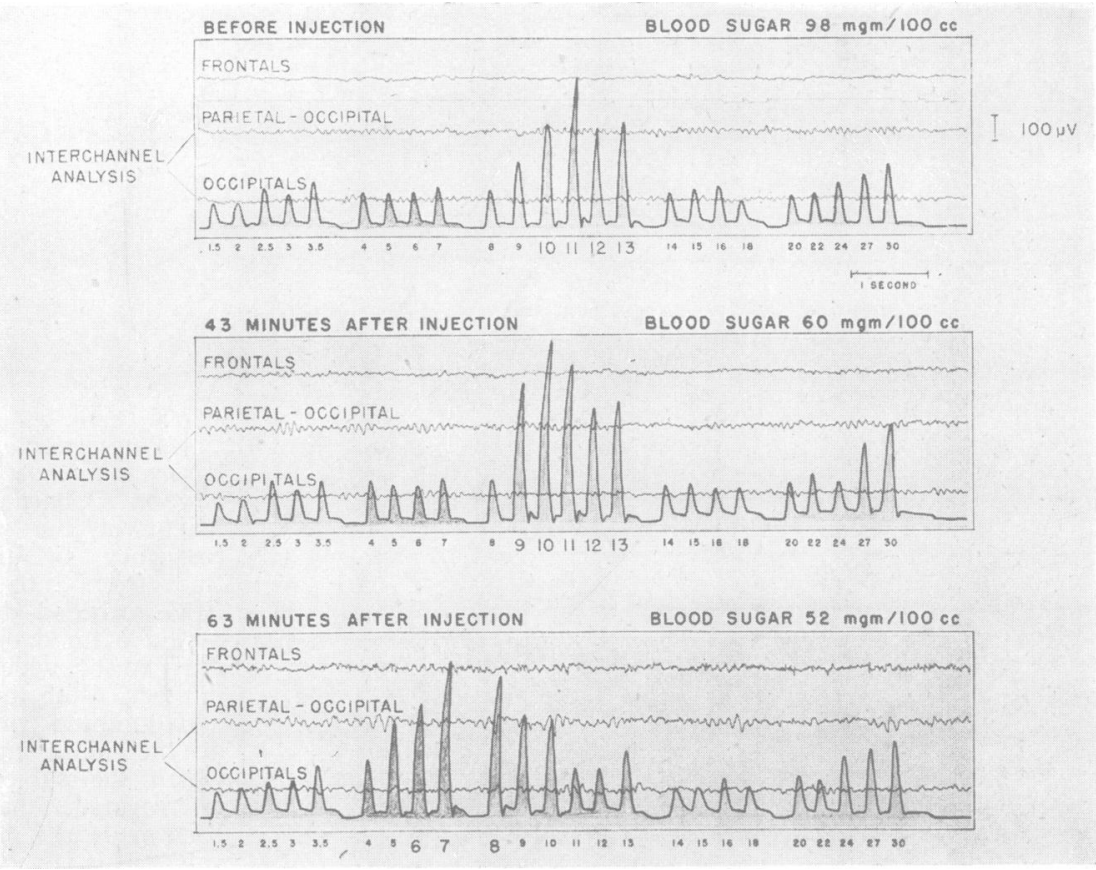

FIG. 6.-Effect of insulin on EEG frequencies. Electro-encephalograms of a

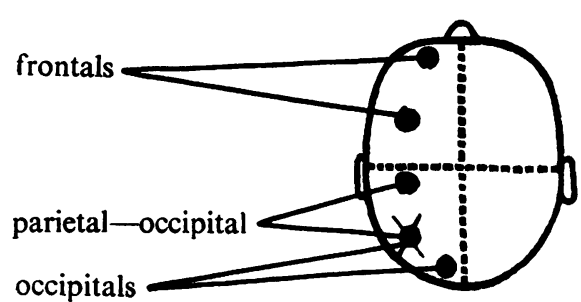
normal man with simultaneous automatic analysis. The electrode placements and connexions were those indicated in the diagram. Interchannel analysis is of the activity out-of-phase between the second and third channel, that is, of an alpha focus at $X$. The frequency represented by each excursion of the analyser's trace is indicated by the number below it.

Analysis shows them to have a frequency usually of 24 to 27 cycles per second.

The second change regularly occurs when the subject becomes unconscious, that is, when he loses contact with his environment and enters the second stage of anæsthesia. Concurrently with this loss of consciousness the fast waves in the EEG disappear and are supplanted by slow waves.

One example of these changes is seen in Fig. 8. In this illustration EEG recordings are shown from three parts of the head, as indicated in the legend accompanying the figure, and the simultaneous analysis of the strong alpha focus in the third channel is superimposed. During the first stage of anæsthesia, while this subject was euphoric, activity was mostly in the fast frequencies from 20 to 27 cycles per second. When he became unconscious, bands of slow waves centering around 2.5 and 6 cycles per second dominated the record.

In this individual the alpha frequencies have been almost extinguished, but it is common to find these persisting even in the second plane of the third stage of anæsthesia. An example is shown in Fig. 9. In this illustration, for clarity only the EEG tracing which is being analysed is reproduced. It is from an alpha focus in the parieto-occipital region. The disappearance of the fast waves when the delta and slow frequencies come in is again seen and the persistence of alpha frequencies is marked.

To illustrate more clearly the sequence of changes in EEG frequencies which accompany shifting levels of consciousness in pentothal anæsthesia a different method of charting has been used. This is seen in the analysis of an alpha focus in another EEG given in Fig. 10. The chart reads from top to bottom, and the width of the white band indicates the spread of frequencies found in the EEG by automatic analysis, plotted every half-minute throughout the whole experiment. The black spots 


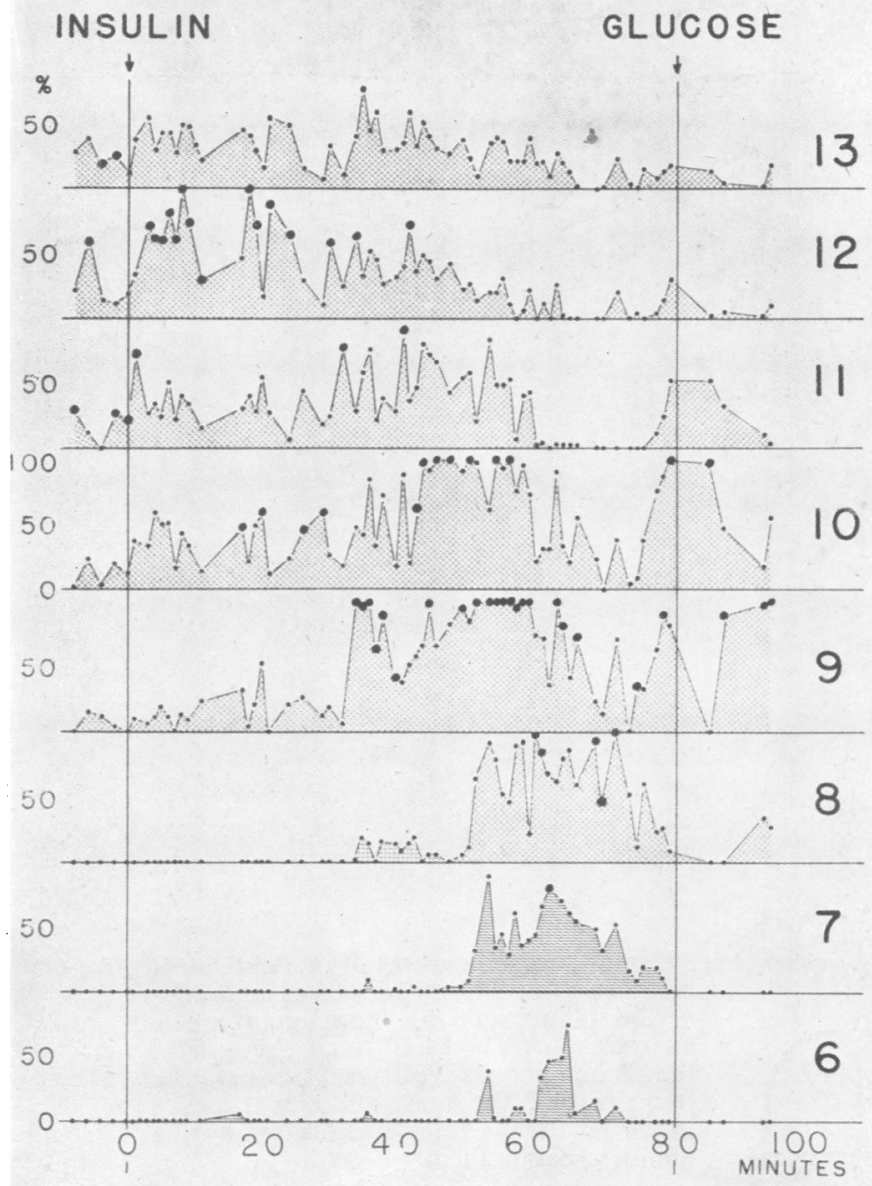

FIG. 7.-Progression of EEG changes in hypoglycamia. Chart depicting the amplitude of the peak at each frequency of the analyser's trace from 6 to $13 \mathrm{c} / \mathrm{s}$ from minute to minute in an EEG recorded during and after the intravenous injection of insulin. This is the same experiment and the same electro-encephalogram as that shown in Fig. 6. represent the actual frequencies present as indicated on the abscissa, and the larger black dot the dominant frequency of each recorded sample. The column on the right indicates the levels of anæsthesia from its induction to the second plane of the third stage, and then the gradual return to a normal state of consciousness.

There are certain quite striking data to be seen in this chart. The development of the fast frequencies in the EEG begins during the first stage, when the cortex is still functioning and when the subject is still conscious. These abnormal fast frequencies are not found when the subject is truly alert, but, as noted above, are evident only when there is some degree of clouding or euphoria. Up to this point all activity in the EEG is in the alpha range. The coincidence of loss of consciousness and the first appearance of slow waves is dramatic, and the fast activity is gone at the same moment. It will be noted that at no time are alpha frequencies absent from the record, although in these cases, when the subject is unconscious, lifting the lids and shining a light directly into the eyes does not block out the activity in the alpha band.

In all cases on emergence from pentothal anæsthesia fast activity returns some time before the slow waves disappear. Neither the EEG changes nor the clinical signs associated with the various planes and stages of anæsthesia are as clear during emergence as they are during induction.

The distribution of frequencies on losis of consciousness from pentothal is different from that found in our record of the subject who fainted, and whose record has been described above. When the EEG frequencies of his record are plotted by the method used in Fig. 10, the distribution is found to be almost entirely within the alpha band until shortly before he faints. Some 4 to 7 cycles per second then appear in the record, and as he faints the alpha disappears for the first time and delta 

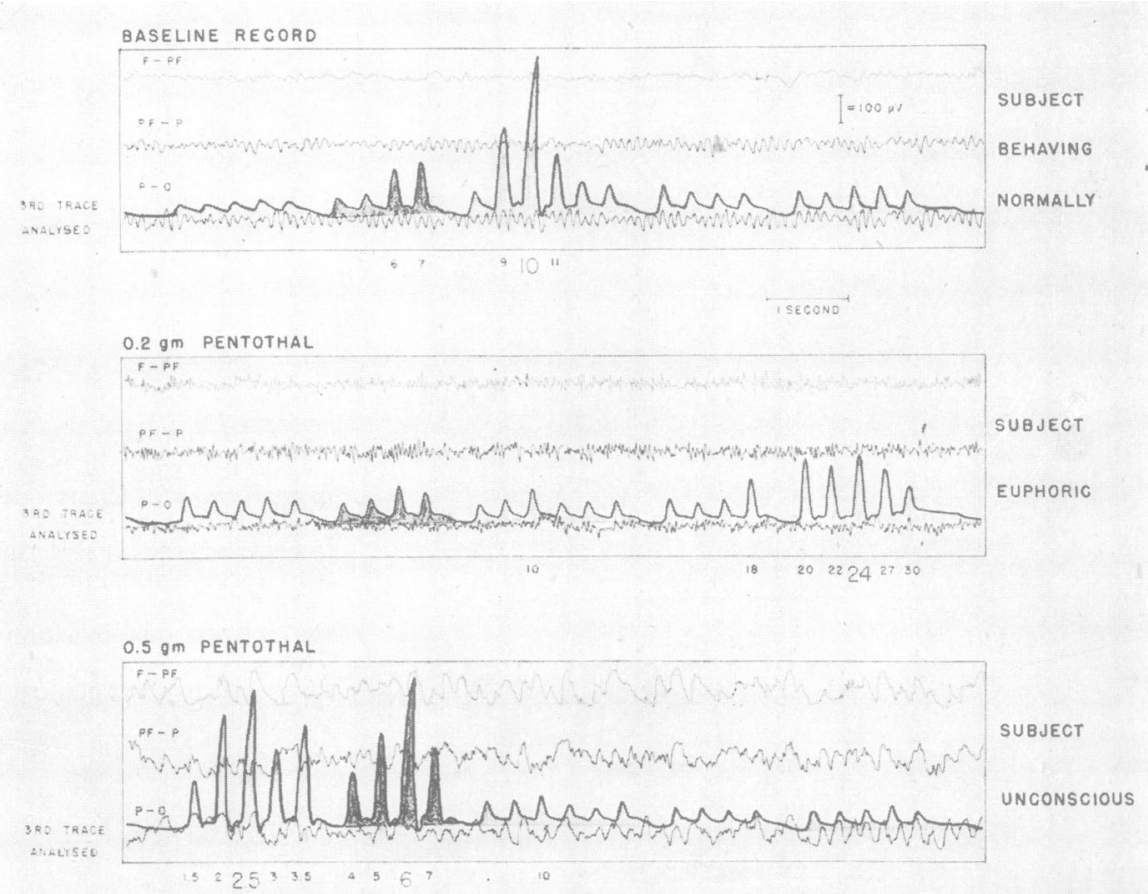

FIG. 8.-Effect of pentothal on EEG frequencies. Electro-encephalograms of one individual

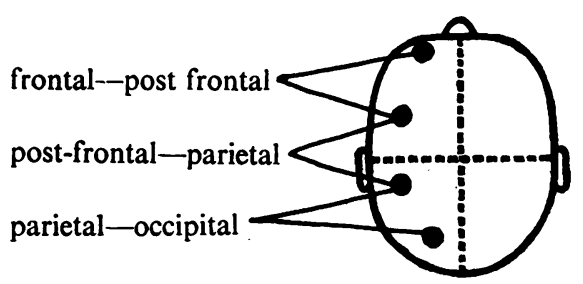
recorded from electrodes placed and connected as indicated by the diagram. The analysis is of the EEG tracing shown in the third channel of each strip. The frequency of the most prominent peaks in the analyser's trace is indicated by the numbers below them.

waves replace it (Fig. 11). The faint lasts less than a minute; on recovery alpha frequencies return and the delta disappears, but a slow band of activity between 4 and 7 cycles per second persists. EEG recording could not be continued for more than a minute following recovery, because of the desirability of moving the subject. At no time was there any EEG activity at frequencies faster than 13 cycles per second.

\section{Discussion}

The striking similarity between the effect on EEG frequencies of inadequate supplies of oxygen and of sugar is understandable on a basis relating these potentials to brain respiration rates, since these two substances play the major roles in cerebral metabolism. That lack of either produces the same effect is in keeping with several known facts, such as that cerebral oxygen uptake is decreased at low levels of blood sugar and increased on administration of sugar (Himwich and others, 1939), and that the action of hypoglycæmia on the EEG can be offset by the inhalation of pure oxygen (Gellhorn and Kessler, 1942).

The data reported here on oxygen lack and on hypoglycæmia at levels where consciousness is retained show a progressive slowing of the alpha rhythm suggestive of a deceleration of the synchronized beat of a uniform neurone population. This view is further supported by the observation that opening the eyes blocks the observed activity in the EEG even when this has slowed to frequencies outside the alpha band. Such observations would be covered by the hypothesis that there is a correlation between the rate of metabolism in the intrinsic cells of the cortex and the relative alpha frequency. 


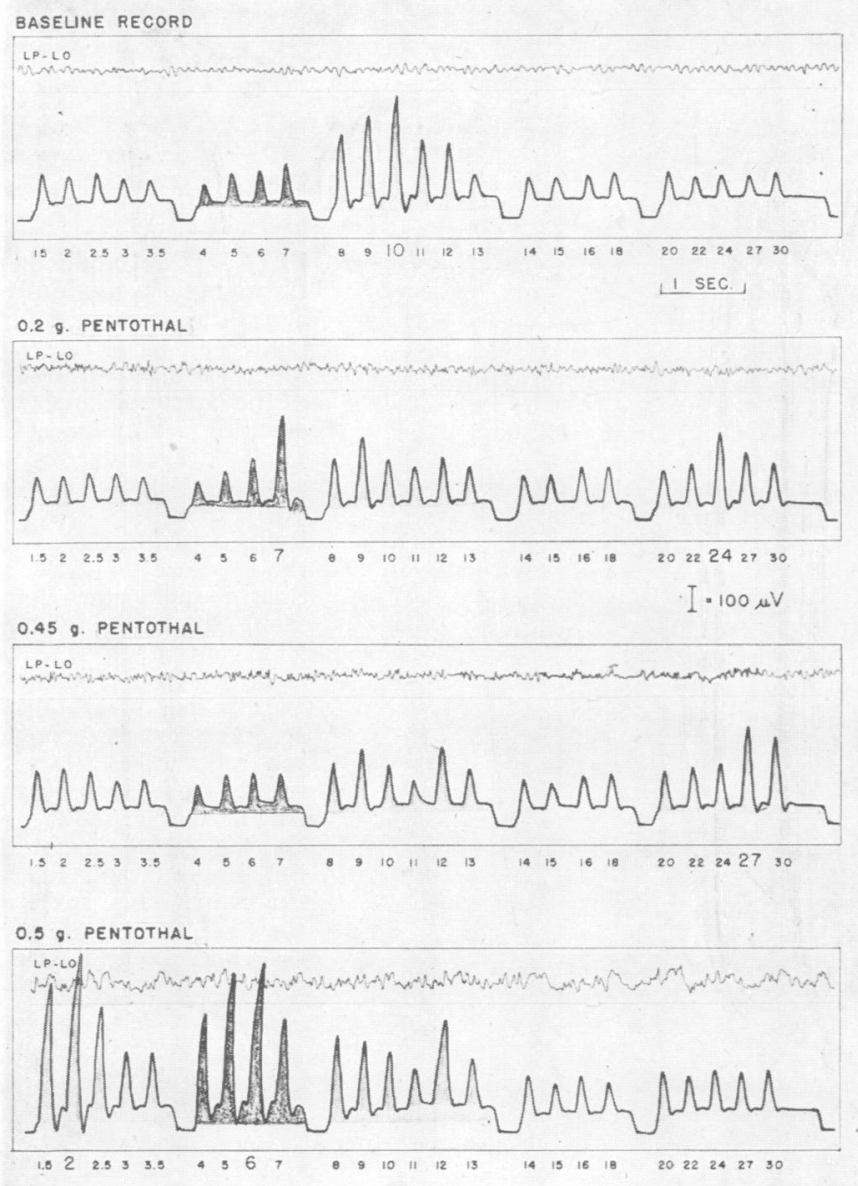

FIG. 9.-Effect of pentothal on EEG frequencies. Electro-encephalograms, with analysis, recorded between two scalp electrodes, one over the parietal and the other over the occipital region of the left hemisphere of an individual before and during pentothal anæsthesia. For clarity only that EEG tracing which is being analysed is reproduced here. The frequency represented by each excursion of the analyser's trace is indicated by the number below it.

That the cortical cells are likely to be implicated before the thalamic nuclei is suggested by the work of, among others, Sugar and Gerard (1938), who, in a comparative study of various regions of the brain (in cats), established that the cortex was more vulnerable to anoxia than the thalamus, and that this paralleled the oxygen requirements determined directly by Dixon and Meyer (1936).

Interpreted in terms of reverberation in thalamocortical circuits one would need to consider not only the thalamic and cortical cell bodies, but the axones and synapses in the neurone chains. Synaptic conduction has proved to be very resistant to anoxia, at least as much so as fibre conduction (Bronk and others, 1938). From studies of fibre conduction in peripheral nerve it is known that the spike potential which is associated with the passage of the nerve impulse is extremely stable to changes in metabolic environment; it is the afterpotentials which are affected by anoxia, and, as Erlanger and
Gasser (1937) have demonstrated, a nerve can be brought by mild asphyxia to a state in which it produces good single spikes but poor negative afterpotentials so that the preparedness of the fibre to receive repetitive impulses is impaired. Such an impairment would clearly affect any process involving the summation of trains of action potentials to produce waves of alpha frequency. Similar conditions apply to the cell bodies : any mechanism relying on summation of repetitive stimuli would result in a rate of discharge dependent not only on the frequency of these impulses but on the rate of oxidative recovery in the neurones under bombardment. Bartley and Bishop (1933), in their original work on responses of the optic cortex, expressed the opinion that cortical cells play a more dominant role than the fibres in the electrical activity. They based this not only on the vulnerability of the cortical potentials to degrees of anæmia and anæsthesia to which fibres would be impervious, 


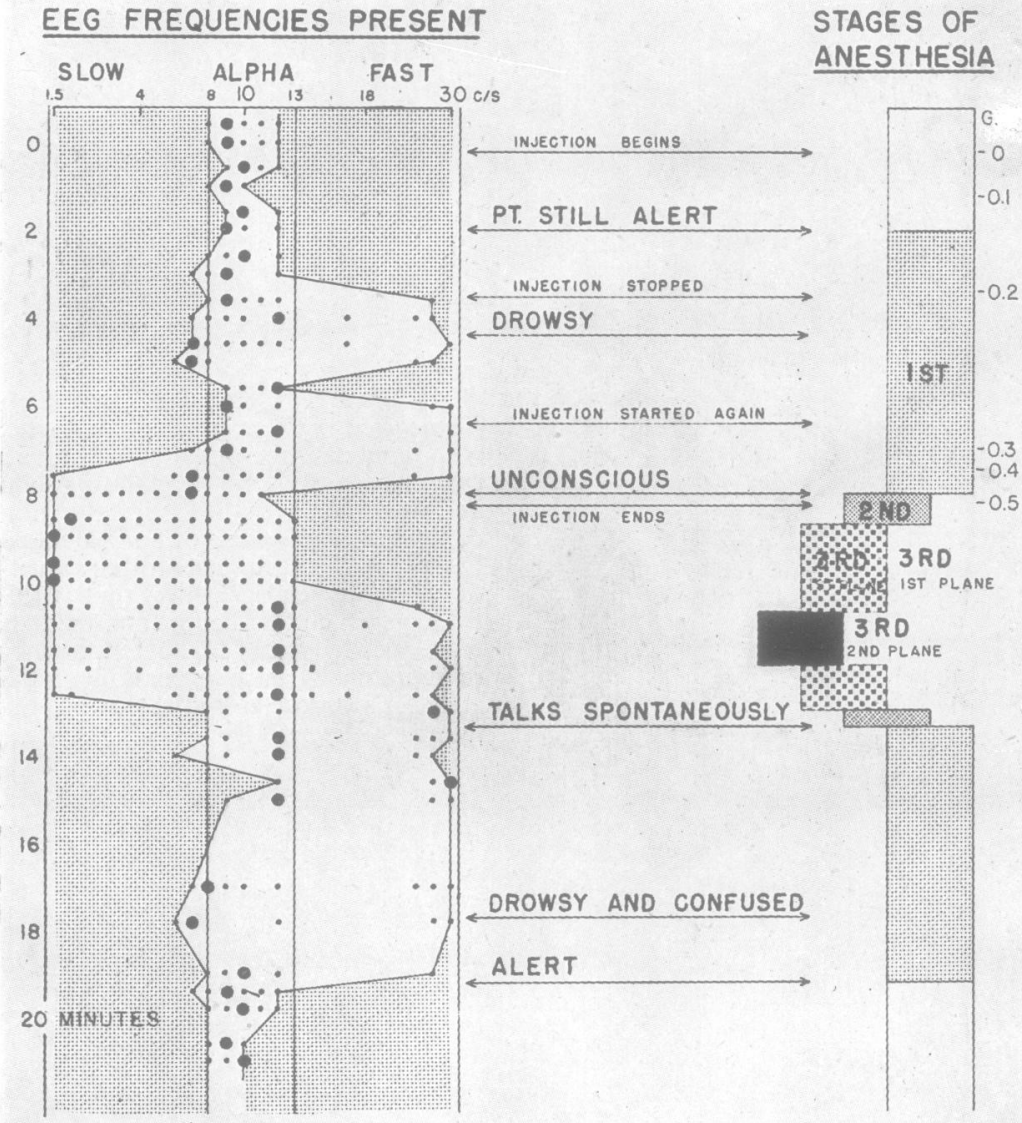

FIG, 10.-The EEG in pentothal anasthesia. Chart showing which frequencies were present in the EEG of an individual throughout a period of anæsthesia induced by pentothal. The experiment begins at the top of the chart and proceeds vertically downwards. Each dof indicates a significant peak in the analyser's trace at the frequency indicated on the abscissa. The heavy dots indicate the dominant frequency of the EEG in each plotted sample. The EEG data are charted at thirtysecond intervals throughout most of the experiment. The concurrent stages of anæsthesia arê charted on the right ō the diagram and follow $\vec{\infty}$ the same time scale a the EEG from the top ob 음 the chart to the bottomi

but also on the magnitude of the potentials recorded, which they thought too great to be accounted for by the summation of fibre potentials in a tissue environment found by them to act as a shunt.

Turning back to the data reported here, one finding would be at variance with this line of interpretation. In the one case illustrated in Fig. 6 there is some activity of beta frequency ; unlike the alpha activity present in the same record this does not slacken in rate as the blood sugar falls, an observation which is difficult to reconcile with the theory that the cortical cell plays the dominant role in frequency determination if these beta frequencies are to be considered as representing intrinsic cortical activity (Chatfield and Dempsey, 1942). However, this is an observation on a single individual and need not be given importance until confirmed.

The data from the pentothal studies are more complex. From biochemical studies of the metabolism of glucose by brain slices the sequence has been found to progress through pyruvate, lactate, glutarate, and other metabolites to oxalacetate. At eảch step two hydrogen atoms are detached by the enzyme action of dehydrogenases. It has been clearly demonstrated in vitro (Quastel, 1939) that the inhibitory action of barbiturates on the oxidation by the brain of glucose, lactate, and pyruvate is by the inactivation of this dehydrogenase activity. On this basis it could be postulated that the initial change to fast frequencies could be due to increased intracellular acidity caused by accumulation of unoxidized acid metabolites (for it is known that lowering the $p \mathrm{H}$ of blood raises the frequency of the alpha rhythm). If this were the mechanism it would appear that a point is reached as the depth of anæsthesia progresses at which the initial effect due to the accumulation of acid metabolites is overwhelmed by the second effect due to the slowing up of the principal chemical reaction caused by the accumulation of its metabolites.

If, on the other hand, the rhythm of the EEG were dependent on reverberation in thalamo-cortical 


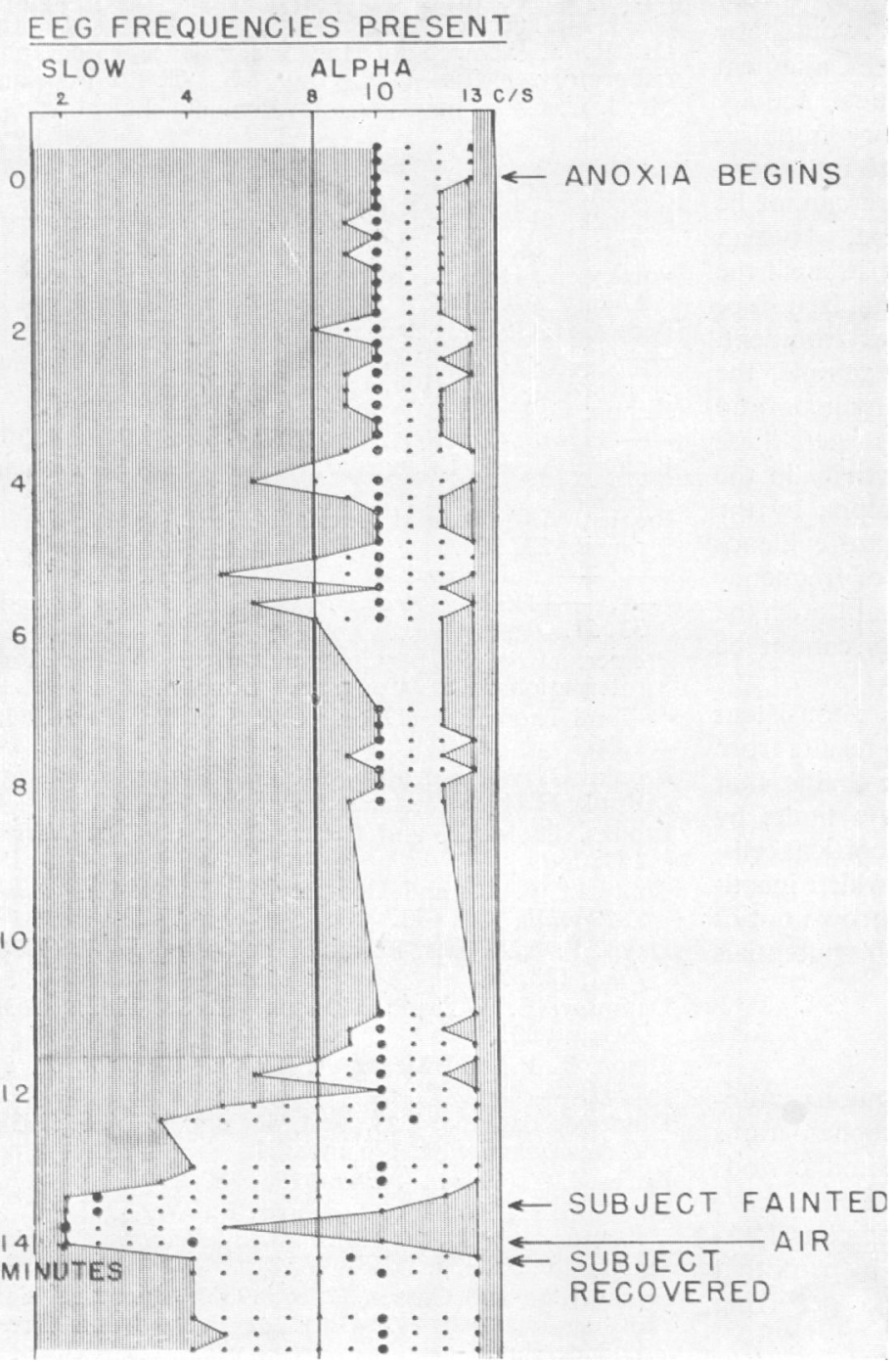

FIG. 11.-The EEG during a faint. Chart showing the distribution of frequencies present in the EEG of an individual throughout a short period of mild anoxia and during a faint. The observations begin at the top of the chart and proceed vertically downwards with time. Each dot indicates a significant peak in the analyser's trace at the frequency indicated on the abscissa. The heavy dots indicate the dominant frequency of the EEG in each plotted sample. This is the same individual and the same electro-encephalogram as in Figs. 4 and 5.

circuits, the two stages of EEG change might represent two loci of attack by the drug. The studies of Etsten and Himwich (1946) have demonstrated the pattern of pentothal anæsthesia to be a descending depression of cerebral oxidations starting with the cerebral hemispheres and progressing down to the lower parts of the brain. During the first stage of anæsthesia (when the EEG shows fast frequencies) there is slight depression of the cortex but it is still the controlling centre. The cortical cells would therefore presumably still be active in any thalamo-cortical circuit. When the subject loses consciousness the cortex has become suppressed and predominant control is by the subcortex. It is at this stage, when the thalamic levels presumably take control, that the slow waves appear in the EEG.

The possibility that the loss of consciousness in pentothal anæsthesia is due to the blocking of afferent impulses from the periphery rather than to a depression of the cortex needs also to be considered. It has been noted above that shining a light into the eyes of subjects under pentohal anæsthesia does not block any EEG rhythms although some are usually of the frequencies defined as alpha ( 8 to 13 cycles per second). Such a concept would introduce the picture of a cortex isolated by the anæsthetic rather than depressed by it. A different mechanism has been advanced by Brooks 
and Eccles (1947) as an extrapolation of their theory of inhibition, namely that narcotics would, by depressing the excitation in the Golgi cells, augment the inhibition, and that lowered cortical activity due to deprivation of incoming sensory impulses would convert excitatory Golgi cells into inhibitors.

Clearly more data are needed. More cannot be said at the present stage of our knowledge. Human electro-encephalography alone cannot answer all the questions, but its role should not be minimized since it is a study of the brain in its natural environment. And it can give some answers: for example, the data from the experiments with mild hypoxia and hypoglycæmia demonstrate that at levels where there is no loss of consciousness the slow activity in the EEG is not a masking of persistent alpha by the eruption of slow activity. The data are evidence for a unitary effect, for a progression of frequency change in the same neurone population, although the anatomical identification of these units cannot be made from experiments of this nature.

In summary, the data reported here are consistent with a postulate that the alpha rhythm results from the repetitive action of cells in neurone chains, that the rate can be modified within certain limits by metabolic changes in the respiration of cortical cells, that it can be disrupted by any agent which inactivates a link in the chain, and that it is thrown out of synchrony by the arrival of action potentials originating as sensory impulses.

\section{Summary}

The results are reported of continuous automatic frequency analysis of electro-encephalograms recorded from human subjects throughout periods of : (1) mild hypoxia without loss of consciousness ; (2) mild hypoglycæmia without loss of consciousness ; (3) loss of consciousness induced by pentothal anæsthesia ; (4) loss of consciousness occurring briefly in one case of fainting.

The data are reviewed in the light of existing hypotheses as to physiological mechanisms, and evidence is presented to show that the observed frequency changes caused by oxygen lack and low blood sugar level are changes in the same neuronal population rather than a replacement by activity from other cell units. The changes in pentothal anæsthesia are more complex and are discussed in comparison with those found in loss of consciousness in the absence of the drug.

The data reported here have been accumulated during the course of several studies in progress in Dr. Stanley Cobb's Department at the Massachusetts General Hospital. The results are the outcome of the collaboration of a team of workers to whom the author is indebted. She especially wishes to thank Dr. J. E. Finesinger and
Dr. H. H. W. Miles of the Department of Psychiatry, Dr. J. H. Tucci of the Department of Anæsthesia, and Dr. R. S. Schwab of the Electro-encephalographic Laboratory. Miss M. N. Gray, Mrs. G. Lothrop, and Mr. J. U. Casby have given invaluable technical help.

\section{REFERENCES}

Adrian, E. D. (1947). Brain, 70, 1.

Baldock, G. R., and Walter, W. G. (1946). Electron. Engng., 18, 339.

Bartley, S. H., and Bishop, G. H. (1933). Amer. J. Physiol., 103, 173.

Berger, H. (1929). Arch. Psychiat., 87, 527. (1934). Ibid., 102, 538.

(1937). Ibid., 106, 165.

Bishop. G. H. (1933). Amer. J. Physiol., 103, 213. (1936). Cold Spr. Harb. Symp. quant. Biol., 4, 305.

Blair, E. A., and Erlanger, J. (1939). Amer. J. Physiol., 126, 97.

Brazier, M. A. B., and Finesinger, J. E. (1944). J. clin. Invest., 23, 303.

,-- and Schwab, R. S. (1944). Ibid., 23, 313.

- - (1945). Arch. Neurol., Psychiat., Chicago, 53,51 .

Bremer, F. (1947). Communication to the First International EEG Congress. London.

and Thomas, J. (1936). C.R. Soc. Biol. Paris., 123, 1256.

Bronk, D. W., Larrabee, M. G., Gaylor, J. B., and Brink, F. (1938). Amer. J. Physiol., 123, 24.

Brooks, C. McC., and Eccles, J. C. (1947). 159, 760.

Chatfield, P. O., and Dempsey, E. W. (1942). Amer. J. Physiol., 135, 633.

Davis, P. A., Davis, H., and Thompson, J. W. (1938). Ibid., 123, 51 .

Dempsey, E. W., and Morison, R. S. (1942). Ibid. 293 and 301.

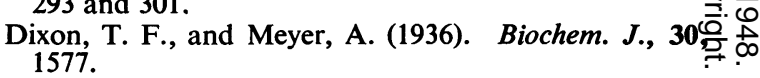

Dusser de Barenne, J. G., and McCulloch, W. S. (1938). J. Neurophysiol., 1, 176 and 364.

Eccles, J. C. (1945). Nature, 156, 680.

Engel, G. L., Romano, J., Ferris, E., Webb. J. P., and Stevens, C. D. (1944). Arch. Neurol. Psychiat., Chicago., 51, 134.

Erlanger, J., and Gasser, H. S. (1937). Electrical signs of nervous activity. Univ. of Pennsylvania Press. Philadelphia.

Etsten, B., and Himwich, H. E. (1946). Anesthesiology, $7,536$.

Fortuyn, J. D., and Jasper, H. H. (1947). Communication to the Seventeenth International Physiological Congress. Oxford.

Gellhorn, E., and Kessler, M. (1942). Amer. J. Physiol., 136, 1 .

Gerard, R. W. (1941). Ohio J. Sci. 41, 160.

and Libet, B. (1940). Amer. J. Psychiat., 96, 1125.

Gibbs, F. A., Williams, D., and Gibbs, E. L. (1940). J. Neurophysiol., 3, 49.

Grass, A. M., and Gibbs, F. A. (1938). Ibid., 1, 521.

Heppenstall, M. E. (1944). J. Neurol. Neurosurg. Psychiat., 7, 112.

Heymans, C., Jourdan, F., and Nowak, S. (1934). R. Soc. Biol. Paris, 117, 470.

Himwich, H. E., Hadidian, Z., Fazekas, J. F., and Hoagland, H. (1939). Amer. J. Physiol., 125, 578. 
Hoagland, H. (1936). Ibid., 116, 604.

(1944). Colloid Chemistry, New York, p. 762. (1933). J. gen. Physiol., 17, 195.

-Rubin, M. A., and Cameron, D. E. (1937). Amer. J. Physiol., 120, 559.

Himwich, H. E., Campbell, E., Fazekas, J. F., and Hadidian, Z. (1939). J. Neurophysiol., 2, 276.

Hodgkin, A. L. (1937). J. Physiol., 90, 211.

Jasper, H. H., Penfield, W., and Droogleever-Fortuyn, J. (1946). Proc. Assn. for Research in Nerv. and Men. Dis. Baltimore. Vol. 26.

Katz, B., and Schmitt, O. H. (1940). J. Physiol., 97, 471.

Lennox, W. G., Gibbs, F. A., and Gibbs, E. L. (1938). J. Neurol. Psychiat., 1, 211.

Libet, B., and Gerard, R. W. (1938). Amer. J. Physiol., 123, 128.

(1938). Proc. Soc. exp. Biol., N.Y., 38, 886.

- -(1939). J. Neurophysiol., 2, 153.
Lorente de Nó, R. (1934). J. Psychol. Neurol., Lpz., 46, 113.

--(1935). Amer. J. Physiol., 113, 505.

(1939). J. Neurophysiol., 2, 402.

McCulloch, W. S. (1947). Fed. Proc., 6, 448.

Morison, R. S., and Dempsey, E. W. (1942). Amer. J. Physiol., 135, 281.

- - and Bassett, D. L. (1945). J. Neurophysiol., 8, 309. Finley, K. H., and Lothrop, G. N. (1943). Ibid., $6,243$.

O'Leary, J. L. (1944). in Bucy. P.C. The Precentral Motor Cortex. University of Illinois Press. Chapter III.

Quastel, J. H. (1939). Physiol. Rev., 19, 135.

Romano, J., and Engel, G. L. (1945). Psychosom. Med.: $7,3$.

Sugar, O., and Gerard, R. W. (1938). J. Neurophysiol., 1,558 .

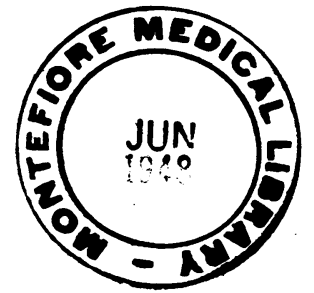

